hep-th/0103071

FIT HE - 01-01

Kagoshima HE-01-2

\title{
Stability of Randall-Sundrum brane-world and tachyonic scalar
}

\author{
Kazuo Ghoroku円 and Akihiro Nakamurał \\ ${ }^{1}$ Fukuoka Institute of Technology, Wajiro, Higashi-ku \\ Fukuoka 811-0295, Japan \\ ${ }^{2}$ Kagoshima University, Korimoto 1-21-35 \\ Kagoshima 890-0065, Japan
}

\begin{abstract}
We address the behaviour of the scalar field with negative mass squared in the five dimensional AdS space where the Randall-Sundrum brane-world is embedded. We point out that the tachyonic scalar allowed in the bulk space destabilizes the embedded brane-world where the cosmological constant is zero. The resolution of this instability is discussed from the viewpoint of AdS/CFT correspondence.
\end{abstract}

\footnotetext{
${ }^{1}$ gouroku@dontaku.fit.ac.jp

${ }^{2}$ nakamura@sci.kagoshima-u.ac.jp
} 


\section{Introduction}

People believe that our four dimensional world would be obtained from the ten or eleven dimensional superstring theory. The D-brane solutions have attracted large attentions of many people, and an interesting geometry has been obtained from the D3-brane of type IIB theory. Near the horizon of the D3-branes, we find the configuration of $A d S_{5} \times$ $S^{5}$. The string theory under this background would describe the four dimensional conformal symmetric Yang-Mills theory on the boundary of $A d S_{5}$ [1, 2, 3, 4] where flat Minkowski space is realized.

On the other hand, a thin three-brane (RS brane) can be embedded in $A d S_{5}$ space [5, 6] at any point of the transverse coordinate, which is interpreted as the energy scale of the conformal field theory on the boundary. A promising picture is to consider this three-brane as our four-dimensional world. This picture gives an alternative to the standard Kaluza-Klein (KK) compactification via the localization of the zero mode of the gravitation [6]. It could also give a new explanation for the problems of the hierarchy between Planck mass and the weak-electromagnetic mass scales. This proposal is distinct from the one given in [7, 8, 9]. Further, it opens a new possible explanation of the smallness of the four dimensional cosmological constant in our world without fine-tuning [10, 11].

In this framework, gravity is defined in the five dimension, but its zero mode is trapped in the brane, then we can observe the usual 4-dim Newton law in the braneworld. Other massive KK modes living in the five dimension are observed as the correction to the Newton law. From the viewpoint of the string theory, the bulk continuum modes are described also by the idea of the AdS/CFT correspondence [12, 14]. The correction to the Newton law can be obtained as the quantum correction from the CFT coupled with the gravitation [14].

Other than the graviton, there would be many kinds of fields in the bulk to be studied since the five dimensional bulk theory would be obtained by the reduction of the ten dimensional IIB theory or eleven dimensional M theory. In any case, we would obtain many number of scalar fields in the reduced five dimensional theory [18, 19]. Many people have tried to derive the domain wall solution, which could be identified with the RS brane in a thin limit, from the reduced supergravity [20, 21. In these approaches, the scalar fields play an important role, so it would be important to know their behaviour in constructing the RS brane(s) in the five dimensional space.

The purpose of this paper is to study the scalar with its wide range of mass allowed in the $A d S_{5}$. In general, negative mass squared is allowed and it is bounded from below $M^{2} \geq-4 / L^{2}$ where $L$ is the radius of AdS space [22]. Our interest is to see how this scalar field can be observed from the brane world embedded in $A d S_{5}$. For $M=0$ case, it is well-known that the zero mode of this scalar is localized in the brane and KK modes are observed as the correction to the zero mode propagator. We extended the analysis to the general case of the scalar mass $M$ according to the technique used in the linearized gravity [12]. In the next section, the solutions of the wave equation are examined to see the localization of some eigenstate of the four dimensional mass 
of the scalar. In the section three, the propagator for this scalar in the bulk space is given by considering appropriate boundary conditions to see the effective two point function of this scalar in the brane-world. We can see that the two different approaches lead to the consistent results. The most important result is that a tachyonic localizedstate appears in the case of bulk tachyonic-scalar, $M^{2}<0$. In section four, an idea for the resolution of this problem is discussed according to the idea of the AdS/CFT correspondence. In the section five, the case of two branes is examined and we find the scalar considered here has nothing to do with the stability of the distance between the two branes. The concluding remarks are given in the final section.

\section{Localized state of the scalar}

Here we start from an effective five-dimensional action which is responsible for the construction of the five dimensional background. It is given in the Einstein frame as,

$$
S_{5}=\frac{1}{2 \kappa^{2}}\left\{\int d X^{5} \sqrt{-G}(R+\Lambda+\cdots)+2 \int d x^{4} \sqrt{-g} K\right\},
$$

where dots denote the contents other than the gravitation, and $K$ is the extrinsic curvature on the boundary. The brane action,

$$
S_{\mathrm{b} 1}=-\frac{\tau}{2} \int d x^{4} \sqrt{-g}
$$

is added to $S_{5}$ and we obtain the 5-dimensional AdS background

$$
d s^{2}=e^{-2|y| / L} \eta_{\mu \nu} d x^{\mu} d x^{\nu}+d y^{2}
$$

where $\tau=6 /\left(L \kappa^{2}\right)$ and $L=\sqrt{6 / \kappa^{2} \Lambda}$ which denotes the radius of AdS space. The coordinates parallel to the brane are denoted by $x^{\mu}$ and $y$ is the coordinate transverse to the brane.

The fields represented by dots in the bulk action are not needed to construct above background $A d S_{5}$ with RS brane. They are however necessary in solving the problem of the cosmological constant without fine-tuning or in constructing the domain wall solution as a thick brane. Here we concentrate our attention on the problem of the stability of the brane-world when we consider the bulk scalar which does not play any role in constructing the background and it is a part of ... in (1). We consider the scalar with mass $M$, and it is denoted by $\Phi$. Then its field equation in the AdS background is given as

$$
\frac{1}{\sqrt{-G}} \partial_{A}\left(\sqrt{-G} G^{A B} \partial_{B} \Phi\right)-M^{2} \Phi=0
$$

where $A, B$ denote five dimensional suffices. This equation can be rewritten as a one dimensional eigenvalue equation similar to the Schrödinger equation as follows. By decomposing as $\Phi=\phi(x) \hat{\psi}(y) e^{3|y| / 2 L}$ and assuming $\eta^{\mu \nu} \partial_{\mu} \partial_{\nu} \phi=m^{2} \phi$, we obtain

$$
\left[-\partial_{z}^{2}+V(z)\right] \hat{\psi}(z)=m^{2} \hat{\psi}(z)
$$




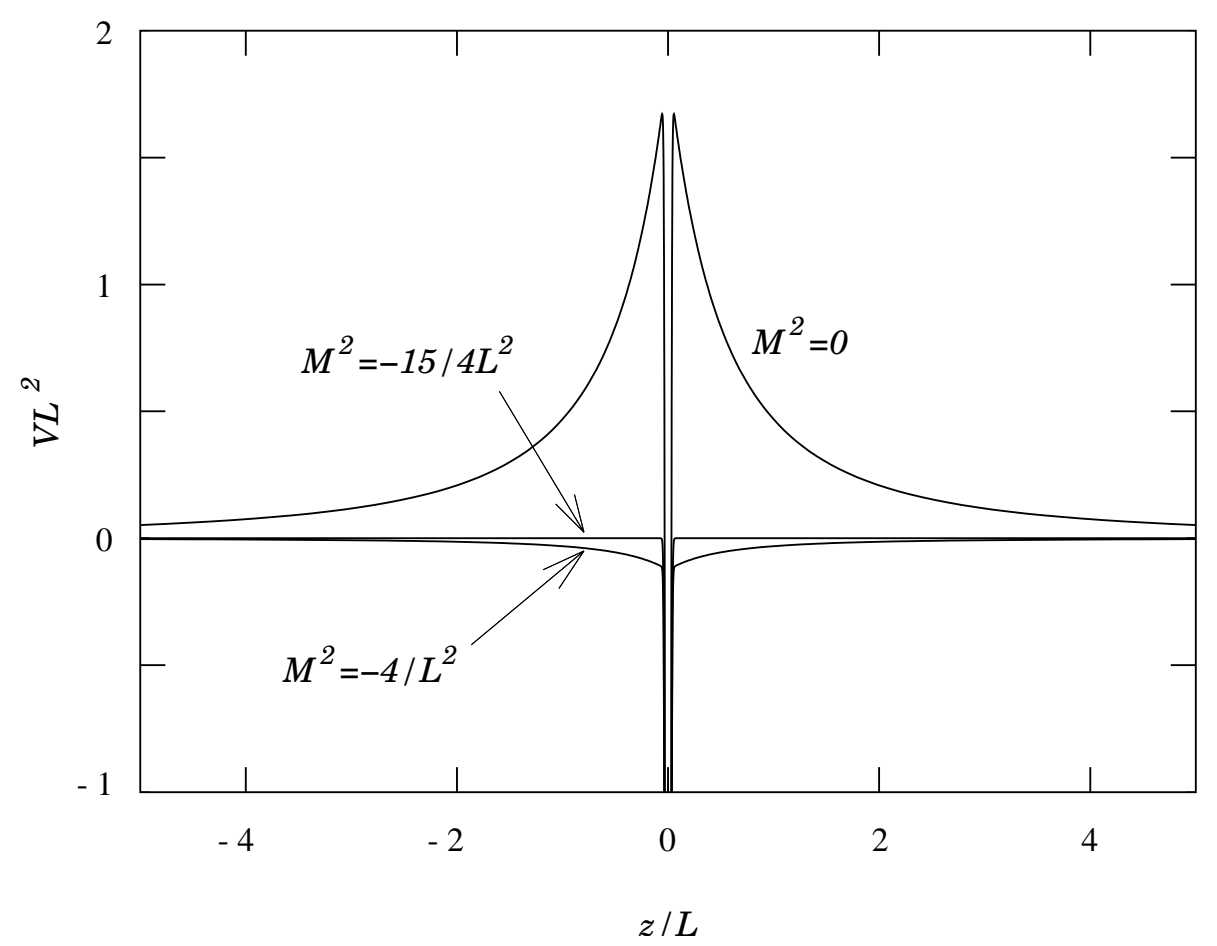

Fig. 1: Shape of the potential $V(z)$

where $z=\operatorname{sgn}(y) L\left(e^{|y| / L}-1\right)$ and

$$
V(z)=\frac{a}{(|z| / L+1)^{2}}-\frac{3}{L} \delta(z), \quad a=\frac{15}{4 L^{2}}+M^{2} .
$$

Equation (5) is regarded as the one dimensional Schrödinger equation with the energy eigenvalue $m^{2}$. For the case of $M=0$, this is equivalent to the one of the graviton. In this case, $a=15 / 4 L^{2}>0$ and the potential has the shape of a volcano (see Fig. 1). This is a necessary condition to localize the zero mode $(m=0)$ in the brane.

In general, the shape of the potential varies with the scalar mass $M$, which is bounded as $M^{2} \geq-4 / L^{2}$ in the AdS background by the positivity of the energy. Within this bound, we can see that the volcano type potential disappears at $M^{2}=-15 / 4 L^{2}$, where $a=0$. So we expect a tachyonic bound state in the brane at least in the region $-15 / 4 L^{2}>M^{2} \geq-4 / L^{2}$. This implies that the RS brane-world with flat background would be unstable if the above tachyonic scalar resides in the bulk.

The eigenvalue of $m^{2}$ for the tachyonic bound state is obtained as follows. Firstly, $m^{2}$ is replaced by $-m^{2}$ in (5) since we are solving this equation in the region $m^{2}<0$. After this replacing, $m$ is considered as a real number. Secondly, we demands that the wave function of the bound state would be normalizable in the sense that the following 
integral with respect to $y$ is finite,

$$
\int d y \sqrt{-G}\left(G^{A B} \partial_{A} \Phi \partial_{B} \Phi+M^{2} \Phi^{2}\right)=\sqrt{-g}\left(g^{\mu \nu} \partial_{\mu} \tilde{\phi} \partial_{\nu} \tilde{\phi}+m^{2} \tilde{\phi}^{2}\right)
$$

where the right hand side provides the effective action for the bound state scalar, which is denoted by $\tilde{\phi}(x)$. It is related to $\phi(x)$ defined previously by

$$
\tilde{\phi}(x)=\sqrt{\int_{0}^{\infty} d y \hat{\psi}^{2} e^{|y| / L}} \phi(x) .
$$

Then the condition of the normalizability is equivalent to the finiteness of the integral in (8). In general, the solution of (5) can be written by the linear combination of two kinds of modified Bessel functions. But the solution here is written by one of them due to the requirement of the normalizability,

$$
\hat{\psi}=N x^{1 / 2} K_{\nu}(m x)
$$

where $x=|z|+L, \nu=\sqrt{4+M^{2} L^{2}}$ and $K_{\nu}$ denotes the second kind of modified Bessel function, which converges at large $m x$. $N$ is a normalization constant.

We need the following boundary condition for this solution at the position of the brane, at $z=0$, due to the $\delta$-function potential,

$$
\left.\partial_{z} \hat{\psi}\right|_{z=0}=-\left.\frac{3}{2 L} \hat{\psi}\right|_{z=0}
$$

This condition leads to the following,

$$
(2+\nu) K_{\nu}(m L)=(m L) K_{\nu+1}(m L)
$$

This equation determines the eigenvalue of $m$.

From the discussion given above, the region to be examined would be restricted to the region $-15 / 4 L^{2}>M^{2} \geq-4 / L^{2}$ or equivalently $1 / 2>\nu \geq 0$ where the volcano type of shape of the potential disappears. However we solve the above equation (11) by extending the region to $\nu \geq 0$. After a numerical research, (i) we can't find any solution for $\nu>2$ expectedly. (ii) At $\nu=2(M=0)$, we find the solution at $m=0$, and this reflects the localization of the zero mode of a massless scalar in the bulk. This is parallel to the localization of the zero mode of the graviton. (iii) In the region $2>\nu>1 / 2$, the potential preserves the volcano shape as in the case of $\nu=2$, so one would expect to find the localization of the zero mode. However, we couldn't find it and the localization of a tachyonic state was found instead. (iv) For $1 / 2 \geq \nu \geq 0$, the localization of a tachyonic state was found expectedly.

As a result, we find only one solution for $2 \geq \nu \geq 0$, and there is no solution for $\nu>2$. The value of $m$ for the localized state changes continuously with $\nu$ as shown in Fig. 2. Namely, $m L$ smoothly increases from 0 to about 1.5 as $\nu$ decreases from

\footnotetext{
${ }^{3}$ Here we notice that $(9)$ is obtained as the solution of (5) where $m$ is replaced by $\mathrm{im}$.
} 


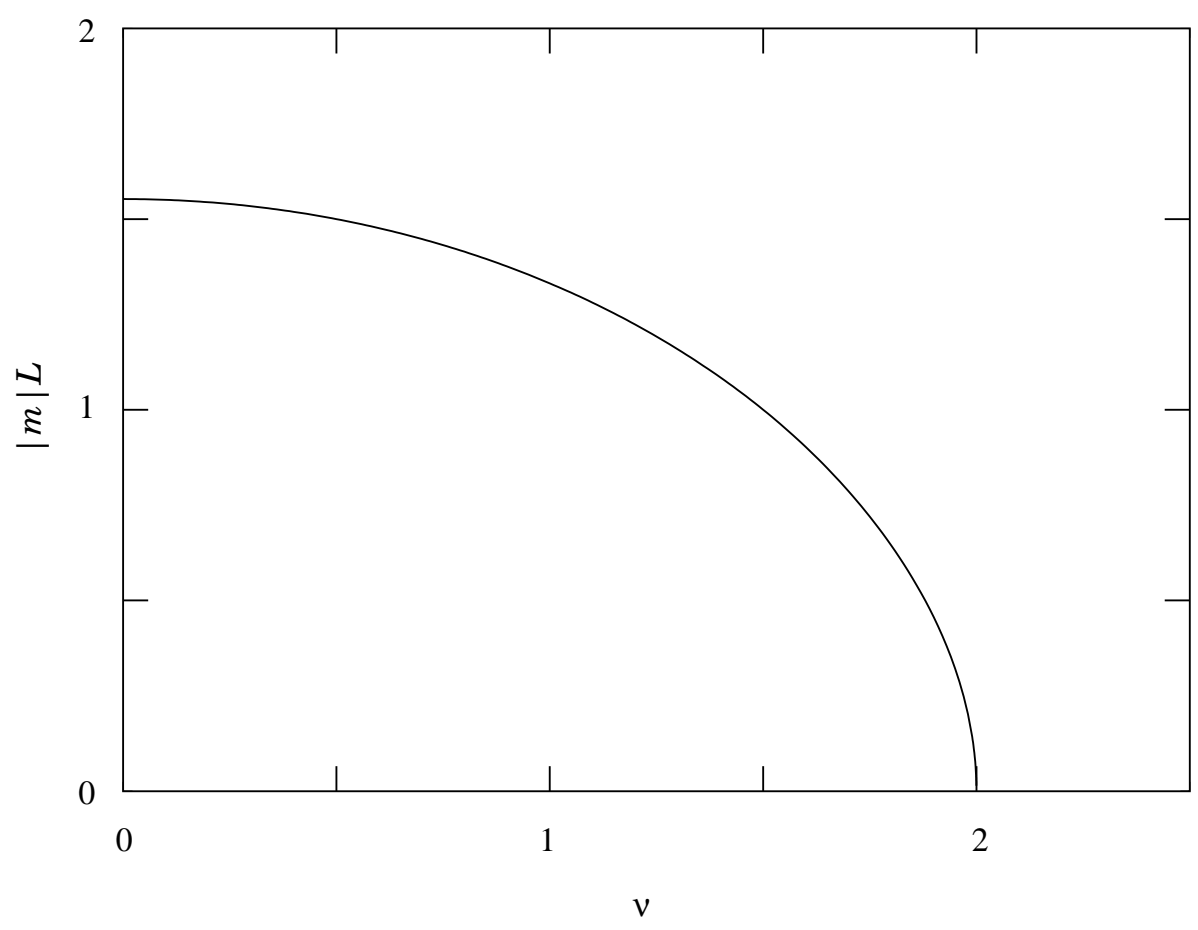

Fig. 2: Location of the tachyon pole vs $\nu$

2 to 0 . This seems to be curious, but we can reassure this point in the next section from the analysis of the five dimensional propagator of the scalar. The existence of only one solution for each $\nu$ is consistent with the $\delta$-functional attractive potential in the eigenvalue equation. The localization of zero mode is realized only for the bulk massless field. The tachyonic localized-state is always seen for the case of the tachyonic bulk scalar, $M^{2}<0$. This can be interpreted as the penetration of the tachyonic state from the bulk into the brane.

\section{Scalar propagator}

According to Ref. [12], the Green function of the scalar considered in the previous section is examined in the $d+1$ dimensional AdS space to see its effective propagator observed in $d$ dimensional flat space of the brane. After obtaining the Green function, we go back to the case of $d=4$. Here we work in the brane background of the following AdS metric,

$$
d s^{2}=\frac{L^{2}}{z^{2}}\left(d z^{2}+d x_{d}^{2}\right) .
$$


We can consider that the brane is located at $z=L$, where $y / L=\ln (z / L)$. The scalar Green function $\Delta_{d+1}\left(X, X^{\prime}\right)$ is defined as

$$
\left(\nabla^{2}-M^{2}\right) \Delta_{d+1}\left(X, X^{\prime}\right)=\frac{\delta^{d+1}\left(X-X^{\prime}\right)}{\sqrt{-G}}
$$

with the Neumann boundary condition,

$$
\left.\partial_{z} \Delta_{d+1}\left(X, X^{\prime}\right)\right|_{z=L}=0
$$

This condition is consistent with the orbifold boundary conditions at the brane.

Equation (13) is rewritten by an ordinary differential equation via following Fourier transform in the $d$ dimensions along the brane,

$$
\Delta_{d+1}\left(x, z ; x^{\prime}, z^{\prime}\right)=\int \frac{d^{d} p}{(2 \pi)^{d}} e^{i p\left(x-x^{\prime}\right)} \Delta_{p}\left(z, z^{\prime}\right)
$$

and the redefinition of the Fourier component, $\Delta_{p}=\left(\frac{z z^{\prime}}{L^{2}}\right)^{\frac{d}{2}} \hat{\Delta}_{p}$. Then the equation for $\hat{\Delta}_{p}$ is obtained as

$$
\left(z^{2} \partial_{z}^{2}+z \partial_{z}+q^{2} z^{2}-\nu^{2}\right) \hat{\Delta}_{p}\left(z, z^{\prime}\right)=L z \delta\left(z-z^{\prime}\right)
$$

where

$$
q=\sqrt{-p^{2}}, \quad \nu=\sqrt{(d / 2)^{2}+L^{2} M^{2}} .
$$

After this, the procedure to obtain the propagator is parallel to [12]. A case that is of particular interest here is that where the arguments of $\Delta_{d+1}$ is on the brane, $z=z^{\prime}=L$. In this case, the propagator is expressed as

$$
\begin{gathered}
\Delta_{d+1}\left(x, L ; x^{\prime}, L\right)=\int \frac{d^{d} p}{(2 \pi)^{d}} e^{i p\left(x-x^{\prime}\right)} \frac{1}{F(q, L, M)}, \\
F(q, L, M)=\frac{d-2 \nu}{2 L}+q \frac{H_{\nu-1}^{(1)}(q L)}{H_{\nu}^{(1)}(q L)} .
\end{gathered}
$$

From this result, some interesting features are observed. Hereafter we consider the case of $d=4$.

(i) First, this is reduced to the form given in [12] for $M=0$ (or $\nu=d / 2$ ), and $\Delta_{d+1}\left(x, L ; x^{\prime}, L\right)$ is separated to the $d$-dimensional massless propagator and the exchange of the Kaluza-Klein states.

$$
\Delta_{d+1}\left(x, L ; x^{\prime}, L\right)=\frac{d-2}{L} \Delta_{d}\left(x, x^{\prime}\right)+\Delta_{K K}\left(x, x^{\prime}\right) .
$$

Here

$$
\partial_{\mu} \partial^{\mu} \Delta_{d}\left(x, x^{\prime}\right)=\delta^{d}\left(x-x^{\prime}\right)
$$




$$
\Delta_{K K}\left(x, x^{\prime}\right)=-\int \frac{d^{d} p}{(2 \pi)^{d}} e^{i p\left(x-x^{\prime}\right)} \frac{1}{q} \frac{H_{\frac{d}{2}-2}^{(1)}(q L)}{H_{\frac{d}{2}-1}^{(1)}(q L)} .
$$

$\Delta_{d}\left(x, x^{\prime}\right)$ represents the localized massless state in the $d(=4)$ dimensional brane. The leading part of the summation of the KK exchanges gives $1 / r^{3}$ potential as in the case of the gravity.

(ii) For $\nu>2$, we can see the massive pole behaviour by expanding $F(q, L, M)$ near small $q L$,

$$
(d / 2-\nu) / L+(1 / 2)(L /(\nu-1)) q^{2}
$$

which implies a pole at $(q L)^{2}=2(\nu-2)(\nu-1)$. This is of course correct for small $q L$, i.e. near $\nu=2$, but we can find a massive pole at large $q L$ where the above approximate formula can not be applied any more. Then (18) and (19) lead to the statement that we can see a massive four dimensional scalar and the corrections from the KK modes to this massive scalar propagator. But it should be noticed that this massive mode is not localized in the brane since the wave function for this mode is not normalizable. This point can be understood from the potential appearing in the bulk wave-equation for the scalar in the fifth dimensional direction. The states of $q^{2}>0$ would decay into large $z$ region outside the wall of the potential, so this state should be regarded as a resonant state with a finite lifetime. This is consistent with the results of [13], where lifetime of the resonant state is explicitly given for small $M^{2}>0$.

We can assure this point explicitly by rewriting $F(q, L, M)$ as

$$
F(q, L, M)=\frac{d-2 \nu}{2 L}+q \frac{J_{\nu}(q L) J_{\nu-1}(q L)-Y_{\nu}(q L) Y_{\nu-1}(q L)+2 i /(\pi q L)}{J_{\nu}(q L)^{2}+Y_{\nu}(q L)^{2}},
$$

and the resonant states are found as the zero points of the real part of $F(q, L, M)$, where their imaginary parts are positive definite by the formula $J_{\nu}(q L) Y_{\nu-1}(q L)-$ $Y_{\nu}(q L) J_{\nu-1}(q L)=2 /(\pi q L)$. This behaviour is seen at any value of $\nu$, and the positions of the zero-points are slightly different near $q=0$. For typical values of $\nu$, at $\nu=1.6,2.0$ and 2.4, $F(q, L, M)$ are shown in both regions of $q>0$ and imaginary $q$ in Fig. 3 and Fig. 4, respectively.

(iii) For $0<\nu<2$, we know that there is a localized state with $q^{2}<0$ from the analysis of the wave-equation. In fact, we can assure the same result also from the propagator given here. The pole in the region $q^{2}<0$ is obtained as a zero point of $F(q, L, M)$ given in (19) by rewriting as $q \rightarrow i q$ in (19), i.e. $F(i q, L, M)=0$, which is written as

$$
(2+\nu) K_{\nu}(q L)=(q L) K_{\nu+1}(q L) .
$$

This is exactly the boundary condition (11), required at the point of brane in solving the wave equation. The pole point of $q L$ varies smoothly from 0 at $\nu=2$ to 1.5 at $\nu=0$ as shown in the previous section. The approximate formula (23) is valid near $\nu=2$ also in this case. In any case, we can see a tachyonic state and KK modes in the brane for $2>\nu \geq 0$. 

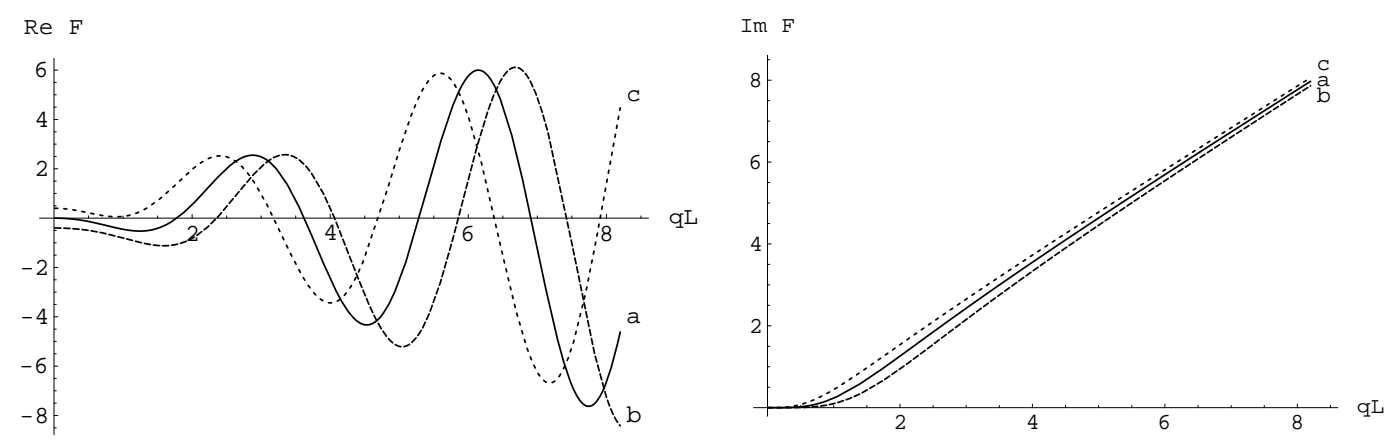

Fig. 3: $F(q, L, M)$ for real $q$. Curves $a, b$ and $c$ represent for $\nu=2.0,1.6$ and 2.4 respectively.

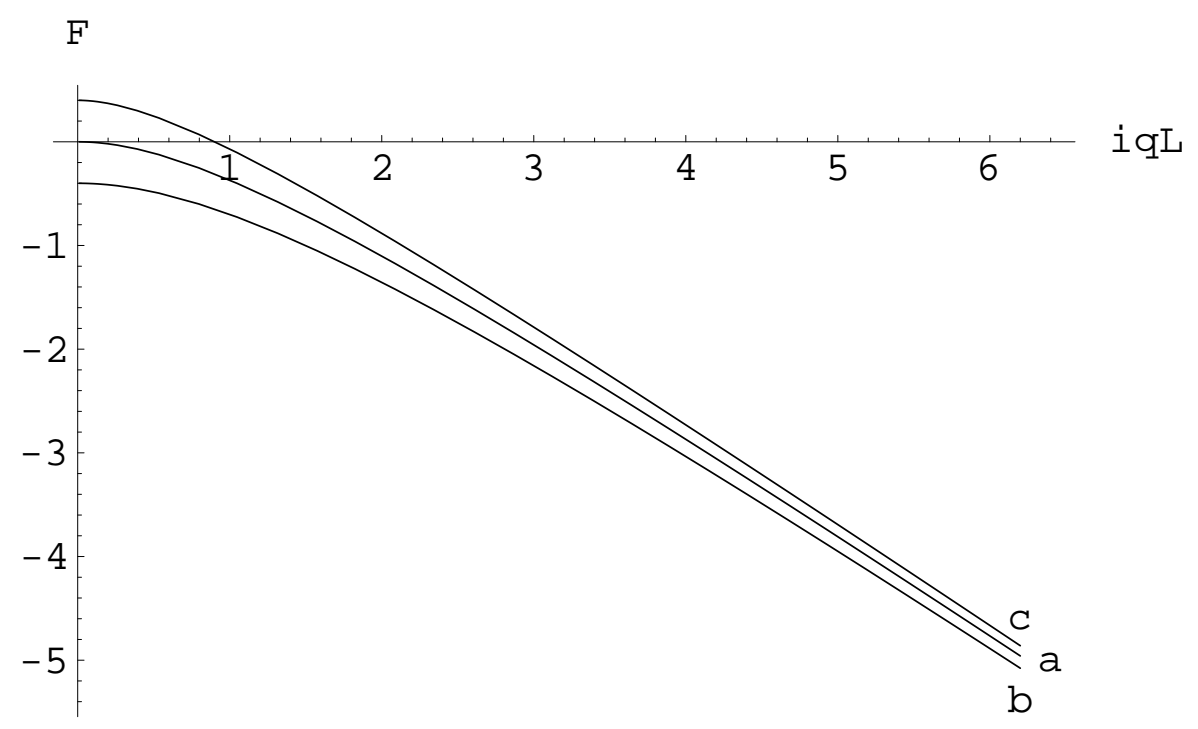

Fig. 4: $F(q, L, M)$ for imaginary $q$. Curves $a, b$ and $c$ represent for $\nu=2.0,1.6$ and 2.4 respectively. 
The signal of the tachyon can be seen in the potential at the long distance as in the case of $\nu=2$, where the massless pole provides the Coulomb potential $1 / r$ at long distance and the next leading $1 / r^{3}$ from the KK-modes. In the case of $\nu<2$, the leading potential comes from the tachyon pole, which behaves as $\cos (|q| r) / r$, and the next leading coming from positive $p^{2}$ region is $1 / r^{3}$. This is easily seen by expanding $F(q, L, M)$ at small $q$ and performing the Fourier transformation of the leading terms. So we can say that the dominant part of the potential at the long distance is given by the exchange of the tachyon.

Then the brane would be unstable since the tachyon is not allowed in the flat four dimensional brane.

We can summarize the above results from the viewpoint of the exchange of $\phi$ fields between two sources. For $\nu=2$, we can see that at large distances on the brane, $\left|x-x^{\prime}\right| \gg L$, the zero mode piece dominates and we reproduce the standard effective action for $d$-dimensional scalar exchange, plus sub-leading corrections from the KaluzaKlein part. This picture is not true for $\nu>2$, and we can not see any Coulomb like behaviour. For $\nu<2$, the tachyon which is allowed in the bulk $A d S_{5}$ penetrates into the brane as a tachyon in the four dimensional brane, and it provides the dominant part of the potential between two charges at long distance. This leads to the instability of the RS brane with flat four-dimensional metric. A simple way to remove this instability is to add the following quadratic term of the scalar $\Phi$ to the brane action $S_{\mathrm{bl}}$,

$$
\int d^{4} x \sqrt{-g} \frac{1}{2} \frac{d-2 \nu}{L} \Phi^{2}
$$

Since this term could cancel out the first term of $F(q, L, M)$ given in (19), which represents the effective quadratic term of the scalar $\Phi$ in the brane, then we obtain the same propagator with the one of $M=0$. Then we can see the localized zero mode for wide range of $\nu$, i.e. for $\nu>1$.

If we adopt the term (26), the boundary condition of the Green function is changed. By taking into account this term with a coefficient independent on the scalar mass $M$, the problem of the localization of a scalar field was also discussed in 23] from a different viewpoint and in a different region of its mass with our analysis. At present, we can't say anything definitely about the meaning of this new term.

More probable resolution of this instability will be to solve the equations of motion for the system of gravity and a scalar with a localized brane-action. It would be a challenging subject to find a solution of Randall-Sundrum type brane from a supersymmetric model induced from the super-string theory. And the action would contain a non-trivial potential and the scalar field would be solved as a non-trivial configuration forming the background [24]. The metric might be solved in the form

$$
d s^{2}=e^{A(y)} \eta_{\mu \nu} d x^{\mu} d x^{\nu}+d y^{2}
$$

and the cosmological constant on the brane can be taken as zero.

In this case, the instability pointed out here would be evaded by the background configuration which control the equations of the fluctuation of the scalar and gravitation. 
In the next section, we discuss how this instability would be resolved from the viewpoint of $\mathrm{AdS} / \mathrm{CFT}$ correspondence. It would give a clue to consider the meaning of the above newly added term and the string induced bulk-action with a scalar.

\section{AdS/CFT correspondence}

The gravitational theory in the background of $A d S_{5}$ is dual to the $4 \mathrm{~d}$ conformal field theory with a cutoff at the position of the $\mathrm{RS}$ brane, so we can replace the five dimensional bulk part by a CFT defined as

$$
S_{\mathrm{CFT}}=\int d^{4} x\left(L_{\mathrm{CFT}}+\Sigma_{i} \lambda_{i} O^{i}\right)
$$

where $O^{i}$ are the composite operators of the fields contained in $L_{\mathrm{CFT}}$ and $\lambda_{i}$ are their corresponding sources which are given as the boundary values of the bulk fields at the brane position. Here we should consider both the gravitation and the scalar on the brane.

Then the effective brane action could be obtained by adding the regularized part of intrinsic curvature 25, 26, 12] and the localized scalar part, $L_{\phi}$, as follows

$$
S_{\mathrm{b} 2}=\int d x^{4} \sqrt{-g}\left\{L_{\text {brane }}+L_{\phi}-\left(\frac{\tau}{2}+b_{0}\right)-b_{2} R-b_{4} R_{2}\right\},
$$

where $b_{0}=-(6 / L) /\left(2 \kappa^{2}\right), b_{2}=-(L / 2) /\left(2 \kappa^{2}\right), b_{4}=2 L^{3} /\left(2 \kappa^{2}\right)$ and

$$
R_{2}=-\frac{1}{8} R_{\mu \nu} R^{\mu \nu}+\frac{1}{24} R^{2}
$$

Since $\tau=(12 / L) /\left(2 \kappa^{2}\right)$, the cosmological constant on the brane, $\tau / 2+b_{0}$, is zero. This is consistent with the Poincare invariant solution. The four dimensional curvature term reflects the localized zero mode of gravity and the curvature squared terms are the correction to the gravity part.

The corrections to $S_{\mathrm{b} 2}$ would be obtained by the CFT part. It is seen by integrating the fields contained in $L_{\mathrm{CFT}}$. In the case of the graviton, this correction has been explicitly shown within one-loop approximation [14, and the correspondence of this correction and the sum up of the KK modes has been assured.

As for the scalar field, it is difficult to give the explicit form of the composite operator and to separate the propagator obtained in the previous section into two parts, the bounded part and KK modes for general $\nu$. Then we don't try to see the correspondence for the quadratic term of $\phi$ here as shown for the graviton. Instead, we use the AdS/CFT correspondence to see the effective action of the scalar $\phi$ for a calculable case. As in [14], we consider $\mathcal{N}=4$ supersymmetric Yang-Mills theory as the CFT which lives on the boundary of the $A d S_{5}$. The conformal dimension of the operator is given by $2+\nu$, so the operator is relevant for $\nu<2$. Here we consider the case of $\nu=0$. 
For $\nu=0$, we can consider the mass term of the adjoint scalar fields, which are denoted by $\left(B_{i}\right)^{2}\left(i=1 \sim 6 N^{2}\right)$, of $S U(N)$ Yang-Mills theory as the corresponding composite operator since it is gauge invariant and has the conformal dimension two. The mass term of the vector would break gauge symmetry, so we do not consider it.

By assuming the availability of the AdS/CFT correspondence, we consider the combined action, $S_{\mathrm{CFT}}+S_{\mathrm{b} 2}$, to obtain the effective potential of $\phi$ with its higher powers. It is easy to obtain the one-loop correction by integrating the fields in CFT. In the case of $\nu=0$, we obtain

$$
V_{0}(\phi)=-m_{0}^{2} \phi^{2}+\frac{6 N^{2}}{64 \pi^{2}} \phi^{2} \ln \frac{\phi}{\mu^{2}},
$$

where $m_{0}$ denotes the tachyon mass obtained above for $\nu=0$ and $\mu$ is some mass scale. The first term is included in $L_{\phi}$ in (29), and $6 N^{2}$ represents the degrees of freedom of the adjoint scalar in CFT. Since $L_{\phi}$ should be obtained from (7) and (8), then the notation $\tilde{\phi}$ should be used instead $\phi$. And $\phi$ of the second term should be written by multiplying $\hat{\psi}(0)$ as $\hat{\psi}(0) \phi$, which represents $\Phi$ on the brane. These points are important when we consider the relative ratio of the two terms and the strength of the coupling of the boundary field and composite operator of CFT. But they are not essential hereafter, and we abbreviate its notation as $\phi$.

Further, we should notice that the second term is proportional to $\phi^{2}$ not to $\phi^{4}$ since $\phi$ couples to the mass term of the CFT scalar (denoted by $O_{0}$ ) as $\phi O_{0}$ not as $\phi^{2} O_{0}$ due to its conformal dimension two. Then the above potential can be rewritten as

$$
V_{0}(\phi)=\frac{6 N^{2}}{64 \pi^{2}} \phi^{2} \ln \frac{\phi}{\hat{\mu}^{2}},
$$

where new scale factor $\hat{\mu}$ is defined as $\frac{6 N^{2}}{64 \pi^{2}} \ln \frac{\mu^{2}}{\hat{\mu}^{2}}=-m_{0}^{2}$. Here, we notice that this potential has the same form, except for its sign, with the one obtained from open string field theory [15, 16, 17] for the tachyon field. This seems curious since there is no definite reason to identify RS brane as D-brane.

If this approximation is available, the above result (32) implies that the scalar field should be shifted to see its stable spectrum from the tachyonic point $\phi=0$ to the minimum point of $V_{0}(\phi)$. However, the value of $V_{0}(\phi)$ at its minimum is negative, so the flat metric in the brane is still unstable if there is no extra term to cancel this new cosmological constant. This implies that we must return to the point where we solve the bulk equations to obtain the background metric, $A d S_{5}$, by taking into account of the non-trivial configuration of the scalar fields with negative mass squared. It would be expected in this case that the stable RS brane in the $A d S_{5}$ background would be obtained by a shift of the scalar. Its fluctuation could be observed as a massive particle around a bottom of its effective potential not a tachyon around the top. A simple example would be shown in a separate paper.

It is an open problem to extend this story to the other value of $\nu>0$ or to the other 
dimensional $A d S$. . Here we obtain a resultant potential term, $\phi^{2} \ln \frac{\phi}{\mu^{2}}$, for the tachyonic scalar $\phi$. This form is slightly different from the one considered in the previous section, (26). The problem related to this point would be discussed in the preparing paper.

\section{Two branes}

Here we consider the model of two branes proposed by Randall and Sundrum [5]. In this case, the distance between two branes in the direction of the fifth coordinate is fixed as finite. So the concept of the localization can't be considered in a strict sense. All modes are confined in the region between the two branes, and $m$ would take discrete values instead. However, the localization of the zero gravitational mode would be important in a sense of the wave-function localized near one brane if our world is not five dimensional one.

A problem of this model is to determine the distance at some definite value as a stable point of the brane system. Several people proposed ideas of the resolutions for this issue by considering the bulk fields [29, 30]. In [29], a scalar field has been introduced with special potentials on the branes in order to fix its boundary values. It can be seen from the effective potential of this scalar zero-mode that there is a stable point at a finite distance between two branes. While the scalar-potential on the branes plays an important role to solve the problem, its origin is obscure.

Here we do not give the answers to these problems. We comment on this problem by considering the scalar without any potential in the branes. The scalar considered in the previous sections is this type of scalar. The strategy is to see the effective potential by integrating the action of the scalar part by substituting the solution of the wave equation in the AdS background with two branes on the boundaries. The equation to be solved is given by $(\mathbb{4}) \sim(\sqrt{6})$ and the boundary conditions, (10) at $z=0$ and

$$
\left.\partial_{z} \hat{\psi}\right|_{z_{c}}=-\left.\frac{3}{2 L\left(z_{c} / L+1\right)} \hat{\psi}\right|_{z_{c}}
$$

at $z=z_{c}$ where the second brane exists.

We give some comments on the solution in this case. The general solution of $\hat{\psi}$ can be written by the linear combination of two independent Bessel functions. The two boundary conditions (10) and (33) determine a relation of two coefficients of the Bessel functions and the four dimensional mass-eigenvalue of the allowable state. The eigenvalues are discrete in this case. So it would be difficult to sum up these discrete states to see the effective potential totally as a function of the distance between two branes. However we can see it easily in our case.

\footnotetext{
${ }^{4}$ The idea of $A d S_{d} / \mathrm{CFT}$ correspondence for RS brane has been discussed in [27] for the case of $d=3 \sim 5$. Also in [28], we can see a related discussion from a different formalism.

${ }^{5}$ Here we use "zero-mode" in the sense that the eigenvalue of the four-dimensional Laplacian is zero in the equation.
} 
The equations to be solved are rewritten as the equations of $\chi(y)$, where it is defined as $\Phi=\phi(x) \chi(y)$. Then the above boundary conditions are written as,

$$
\left.\partial_{y} \chi\right|_{y_{1}}=\left.\partial_{y} \chi\right|_{y_{2}}=0
$$

where $y_{1}(=0)$ and $y_{2}$ are the positions of the two branes. On the other hand, the scalar part of the action can be written as follows by using its equation of motion in the AdS background;

$$
\begin{aligned}
S_{\Phi}^{\text {classical }}= & -\frac{1}{2} \int_{y_{1}}^{y_{2}} d y \int d^{4} x \sqrt{-G}\left(G^{A B} \partial_{A} \Phi \partial_{A} \Phi+M^{2} \Phi^{2}\right) \\
= & -\frac{1}{2} \int_{y_{1}}^{y_{2}} d y \int d^{4} x \partial_{\mu}\left(\sqrt{-G} G^{\mu \nu} \Phi \partial_{\nu} \Phi\right) \\
& -\left.\frac{1}{2} \int d^{4} x\left(\sqrt{-G} G^{y y} \Phi \partial_{y} \Phi\right)\right|_{y_{1}} ^{y_{2}} .
\end{aligned}
$$

The first term vanishes since it is total derivative and the second term vanishes due to the boundary conditions (34). Then we can see $S_{\Phi}^{\text {classical }}=0$ so that the scalar considered here has nothing to do with the stabilization of two branes at some finite distance.

As in the case of [29], if some boundary terms of the scalar are added to the brane actions, then the boundary conditions are changed. In this case, $S_{\Phi}^{\text {classical }} \neq 0$. So we must solve the mass spectrum allowed between two branes and they are summed up to obtain the effective potential as a function of the distance between branes. We are now preparing the paper of this issue.

\section{Concluding remarks}

When we consider some supersymmetric five dimensional theory to construct the brane as a soliton or a domain wall, we must aware about the contents of the theory even if it was not used to solve the equations responsible for the background. It would be important to study the behaviours of various fields in the background obtained as a classical solution. Here we have examined the scalar field in the AdS background, in which the Randall-Sundrum three-brane(s) is (are) embedded. The mass-squared of the scalar is extended to the allowable negative value in the $A d S_{5}$ background. We found a localization-mode of the scalar, which has negative mass-squared in the bulk, on the brane, and this localized mode is also tachyonic in the four dimensional braneworld. This fact implies that the three-brane with flat space would be unstable since a tachyonic scalar is living in this brane world. Other continuous tachyonic-modes would not cause any instability of the three brane since they should be considered as the bulk tachyonic scalar being allowed in $A d S_{5}$.

It would be possible to consider several ways to evade this problem of the instability of the brane. (i) One way is to improve the brane-action by adding a boundary potential of the scalar such that it cancels the tachyonic mass of the localized state. As a result, 
we could find a massless localized scalar on the brane. But this procedure seems to be artificial since the boundary potential is added by hand. (ii) Second way is to solve the bulk equations by assuming a non-trivial configuration for the tachyonic scalar, and we try to find a solution which forbids the tachyonic localized state. Or we should obtain a brane solution which allows tachyonic localized-scalar. In this approach, it would be necessary to know the fully corrected, complicated potential for the scalar. (iii) As a third possibility to resolve this problem, we have examined the effects of the bulk on the brane from the viewpoint of AdS/CFT correspondence. For $\nu=0$, we find an interesting scalar potential which can be related to this problem, but we could not find a general clue to resolving the problem in this direction. To get some definite conclusion in this approach, we should examine the properties of many more bulk-operators.

We will discuss on these points in the future paper. We have also examined the role of the tachyonic scalar in the case of two branes, but we find that this scalar gives no effect to the problem of determining the stable point between two branes.

Acknowledgement: We wish to thank Drs. S. Dubovsky and S. Shatashvili for giving us useful informations and references.

\section{References}

[1] J. Maldacena, Adv. Theor. Math. Phys. 2 (1998) 231, (hep-th/9711200).

[2] S.S. Gubser, I.R. Klebanov, and A.M. Polyakov Phys. Lett. B 428 (1998) 105, (hep-th/9802109).

[3] E. Witten, Adv. Theor. Math. Phys. 2 (1998) 253, hep-th/9802150).

[4] A.M. Polyakov, Int. J. Mod. Phys. A14 (1999) 645, (hep-th/9809057).

[5] L. Randall and R. Sundrum, Phys. Rev. Lett. 83 (1999) 3370, (hep-ph/9905221).

[6] L. Randall and R. Sundrum, Phys. Rev. Lett. 83 (1999) 4690, (hep-th/9906064).

[7] N. Arkani-Hamed, S. Dimopoulos, and G. Dvali, Phys. Lett. B429 (1998) 263, (hep-ph/9803315).

[8] L. Antoniadis, N. Arkani-Hamed, S. Dimopoulos and G. Dvali, Phys. Lett. B436 (1998) 257, (hep-ph/9804398).

[9] L. Antoniadis, Phys. Lett. B246 (1990) 377.

[10] N. Arkani-Hamed, S. Dimopoulos, N. Kaloper and R. Sundrum, Phys. Lett. B480 (2000) 193, (hep-th/0001197). 
[11] S. Kachru, M. Schulz and E. Silverstein, Phys. Rev. D62 (2000) 045021, (hep-th/0001206).

[12] S.B. Giddings, E. Katz and L. Randall, JHEP 03 (2000) 023, (hep-th/0002091).

[13] S.L. Dubovski, V.A. Rubakov and P.G. Tinyakov, Phys. Rev. D62 (2000) 105011, (hep-th/0006046).

[14] M.J. Duff and J. T. Liu, Phys. Rev. Lett. 85 (2000) 2052, (hep-th/0003237).

[15] A.A. Gerasimov and S.L. Shatashvili, JHEP 034 (2000) 0010, (hep-th/009103)

[16] D. Kutasov, M. Marino and G. Moore, JHEP 045 (2000) 0010, (hep-th/009148)

[17] J.A. Minahan and B. Zwiebach, JHEP 029 (2000) 09, (hep-th/000823)

[18] M. Pernici, K. Pilch and P. van Nuiuwenhuizen, Nucl. Phys. B259 (1985) 460.

[19] K. Behrndt and M. Cvetic, Phys. Rev. D61 (2000) 101901, (hep-th/0001159).

[20] K. Behrndt and M. Cvetic, Phys. Lett. B475 (2000) 253, (hep-th/9909058).

[21] M.J. Duff, J.T. Liu and K.S. Stelle, "A supersymmetric Type IIB RandallSundrum realization", hep-th/0007120.

[22] P. Breitenlohner and D.Z. Freedman, Ann. Phys. 144 (1982) 249; Phys. Lett. B115 (1982) 197.

[23] M. Mintchev and L. Pilo, "Localization of Quantum Fields on Branes", hep-th 10007002.

[24] O. DeWolfe, D.Z. Freedman, S.S. Gubser and A. Karch, Phys. Rev. D62 (2000) 046008, (hep-th/9909134).

[25] M. Henningson and K. Skenderis, JHEP 07 (1998) 023, (hep-th/9806087).

[26] V. Balasubramanian and P. Kraus, Commun. Math. Phys. 208 (1999) 413, (hep-th/9902121).

[27] L. Anchordoqui, C. Nunez and K. Olsen, JHEP 10 (2000) 050, (hep-th/0007064).

[28] S.Nojiri, S. Odintsov and S. Zerbini, Phys. Rev. D62, (2000) 064006, (hep-th/0001192).

[29] W.D. Goldberger and M.B. Wise, Phys. Rev. Lett. 83 (1999) 4922, (hep-ph /9907447).

[30] M. A. Luty and R. Sundrum, Phys. Rev. D62 (2000) 035008, (hep-th/9910202). 